\title{
Fault Detection and Diagnosis for Non-Gaussian Singular Stochastic Distribution Systems
}

\author{
Shi Jing1 \\ Department of Electrionics and Information Engineering \\ Xianyang Vocational Technical College \\ Xianyang,China \\ 258587000@qq.com
}

\section{Du Peng2}

Department of Electrionics and Information Engineering Xianyang Vocational Technical College

Xianyang,China

285639424@qq.com

\begin{abstract}
Fault detection and diagnosis (FDD) for singular stochastic distribution control (SDC) systems via the output probability density functions(PDFs) have been discussed. The PDFs can be approximated via square-root B-spline expansion,and expansions to represent the dynamics weighting systems between the system input and output
\end{abstract}

\section{INTRODUCTION}

Fault detection and diagnosis (FDD) are important research area for improving control systems reliability. Many effective methods have been presented in the past two decades for stochastic system.Up to now,most of the existing FDD algorithms have only been concerned with Gaussian distribution system. Howere, nonlinearity may lead to non-Gaussian output,where mean and variance of the system output are insufficient to characterize their statistical behavior precisely ([11],[12],[13]). As such ,there a new FDD algorithms is need to develop, which can be applied to the stochastic system subjected to random parameter. It is noted that if only output PDFs can be measured, most FDD approaches are invalid. Now therefore, an observer-based FDD methods have been presented in reference([3],[7],[11],[14],[15],[16], and reference therein) to detect and diagnose the fault for non-Gaussian SDC system. Whereas, for,few literatures have been reported to the FDD problem for singular non-gaussian stochastic distribution control systems . This forms the main purpose of the current work.

This paper is organized as follows. In Section 2, the output PDFs expansion and the nonlinear weight dynamic are established to formulate the FTC problem. In Section 3, the FTC filtering is designed to compensate or reject fault. The concluding remarks are presented in Section 4.

Notations: * denotes the elements below the main diagonal of a symmetric block matrix. I is the identity matrix with appropriate dimensions. $\|\bullet\|$ denotes the Euclidean norm vector in $\mathbf{R}^{\mathbf{n}} \cdot \operatorname{diag}\{\ldots\}$ stands for the block diagonal matrix. A- 1 represents the inverse of

\author{
Qu Yi3 \\ College of Electrical and Information Engineering \\ Lanzhou University of Technology \\ Lanzhou,China \\ 1071570207@qq.com
}

PDFs. an novel fault detection and diagnosis algorithm is presented using the parameter-updating. Finally,the simulation result is included to show that satisfactory robustness and closed-loop performance can be achieved.

Keywords-probability density fuctions;singular stochastic distribution;control;fault detection;diagnosis matrix A. $\operatorname{tr}$ (A) denotes the trace of matrix A. AT refers to the transpose of vector $\mathrm{x}$ or matrix A.

\section{PROBLEM FORMULATION AND PRELIMINARIES}

Consider a continuous-time dynamic stochastic distribution systems where $\mathrm{u}(\mathrm{t}) \in \mathrm{Rm}$ is the control input, $\mathrm{y}(\mathrm{t}) \in[\mathrm{a}, \mathrm{b}]$ represents the system output,and $\mathrm{F}$ is the fault to be compensated or rejected,a typical example of which is an actuator fault.At any time $t$, the probability of output $\mathrm{y}(\mathrm{t})$ lying inside $[\mathrm{a}, \mathrm{b}]$ can be described as

$\sqrt{\gamma(z, u(t), F)}=\sum_{i=1}^{n} v_{i}(u(t), F) b_{i}(z)+\omega_{0}(z, u(t), F)$

where $v_{i}(u(t), F)(i=1,2, \ldots, n)$ are the corresponding weights,$b_{i}(z)(i=1,2, \ldots, n)$ are a pre-specified basis function, and $\omega_{0}(\mathrm{z}, \mathrm{u}(\mathrm{t}), \mathrm{F})$ stands for the model uncertainty or the error,which is supposed to satisfy $\left|\omega_{0}(\mathrm{z}, \mathrm{u}(\mathrm{t}), \mathrm{F})\right| \leq \delta_{0}$, $\delta_{0}$ is a known positive constant.

Denote

$B_{0}(z)=\left[b_{1}(z) \quad b_{2}(z) \cdots b_{n-1}(z)\right]$

$V(t)=V(u(t), F)=\left[v_{1}(u(t), F) \quad v_{2}(u(t), F) \cdots v_{n-1}(u(t), F)\right]^{T}$ and

$\Lambda_{1}=\int_{a}^{b} B^{T}(z) B(z) d z, \Lambda_{2}=\int_{a}^{b} B^{T}(z) b_{n}(z) d z, \Lambda_{3}=\int_{a}^{b} b_{n}^{2}(z) d z \neq 0, \Lambda_{0}=\Lambda_{1} \Lambda_{3}-\Lambda_{2}^{T} \Lambda_{2}$ the following model is given:

$$
\sqrt{\gamma(z, u(t), F)}=B(z) V(t)+h(V(t)) b n(z)+\omega(z, u(t), F)
$$

where

$$
\begin{aligned}
& B(z)=B_{0}(z)-\frac{\Lambda_{2}}{\Lambda_{3}} b_{n}(z) \\
& h(V(t))=\frac{1}{\Lambda_{3}}\left[-\Lambda_{2} V(t)+\sqrt{\Lambda_{2}(t)-V^{T}(t) \Lambda_{0} V(t)}\right]
\end{aligned}
$$

From the boundedness of $\omega_{0}(\mathrm{z}, \mathrm{u}(\mathrm{t}), \mathrm{F})$, it can be assumed 
that $|\omega(\mathrm{z}, \mathrm{u}(\mathrm{t}), \mathrm{F})| \leq \delta$ holds for all $\{\mathrm{z}, \mathrm{u}(\mathrm{t}), \mathrm{F}\}$, where $\delta$ is a known positive constant.

In this paper the nonlinear dynamic model will be considered as follows

$$
\left\{\begin{array}{l}
E \dot{x}(t)=A x(t)+G g(x(t))+D u(t)+F \\
V(t)=C x(t)
\end{array}\right.
$$

where $\mathrm{x}(\mathrm{t}) \in \mathrm{R}^{\mathrm{m}}$ is the unmeasured state, and $\mathrm{A}, \mathrm{G}, \mathrm{D}, \mathrm{C}$ represent the known parametric matrices of the dynamic part of the weight system, In fact, these matrices can be obtained either by physical modeling or the scaling estimation technique described in [1] and [6]; $\mathrm{E} \in \mathrm{R}^{\mathrm{m} \times \mathrm{m}}$ is a known singular matrix,i.e., $\operatorname{rank}(E)=r<m ; g(x(t)) \in R^{m}$ is a nonlinear vector function that represents the nonlinear dynamics of the weight model and is supposed to satisfy $\mathrm{g}(0)=0$.

The following network can be used to approximate the continuous unknown function $\mathrm{F}(\mathrm{t}):=\mathrm{F}(\mathrm{x}, \mathrm{u})$

$$
F(x, u)=T W S(x, u)+\theta(x, u)
$$

where $\mathrm{T}$ is given matrix, $\mathrm{W}$ is the ideal weight matrix, $\theta(\mathrm{x}, \mathrm{u})$ is a neural network approximation error, $\mathrm{S}(\mathrm{x}, \mathrm{u})$ is the basis function. Since the state $\mathrm{x}$ is immeasurable,then the output of neural network can be expressed as

$$
\hat{F}(\hat{x}, u)=T \hat{W} S(\hat{x}, u)
$$

where $\hat{x}(t)$ is the estimated state, $\hat{W}_{1}$ is an estimated matrix

\section{FAULT DETECTION AND DIAGNOSIS VIA OUTPUT PDFS}

\section{A. Observer-based fault detection}

Since the measured informance is the output probability distribution, in order to detect the fault based on the changes of output PDFs, the following full-order observer is applied to detect the fault.

$$
\left\{\begin{array}{c}
E \dot{\hat{x}}(t)=A \hat{x}(t)+G g(\hat{x}(t))+D u(t)+L \varepsilon(t) \\
\varepsilon(t)=\int_{a}^{b} \sigma(z)[\sqrt{\gamma(z, u(t), F)}-\sqrt{\hat{\gamma}(z, u(t), F)}] d z \\
\sqrt{\hat{\gamma}(z, u(t), F)}=B(z) C \hat{x}(t)+h(C \hat{x}(t)) b_{n}(z)
\end{array}\right.
$$

where $\hat{x}(t)$ is the estimated state, $\mathrm{L} \in \mathrm{R}^{\mathrm{m} \times \mathrm{p}}$ is the gain to be determined and the rsidual $\varepsilon(\mathrm{t})$ is formulated as an integral of the difference between the measured PDFs and the estimated ones, $\sigma(\mathrm{z}) \in \mathrm{R}^{\mathrm{m} \times \mathrm{m}}$ can be regarded as a pre-specified weight vector lying $[\mathrm{a}, \mathrm{b}]$ and makes the integration simple or adjust the scale of $\varepsilon(t)$.

By defining $e(t)=x(t)-\hat{x}(t) \quad, \quad \tilde{g}(t)=g(x(t))-g(\hat{x}(t)) \quad$, $\tilde{h}(t)=h(C x(t))-h(C \hat{x}(t))$, the estimation error system can be described

$$
E \dot{e}(t)=\left(A-L \Gamma_{1}\right) e(t)+G \tilde{g}(t)-L \Gamma_{2} \tilde{h}(t)-L \Delta(t)+F
$$

where

$$
\begin{aligned}
& \Gamma_{1}=\int_{a}^{b} \sigma(z) B^{T}(z) E d z, \Gamma_{2}=\int_{a}^{b} \sigma(z) b_{n}(z) E d z, \Delta(t)=\int_{a}^{b} \sigma(z) \omega(z, u(t), F) d z \\
& \text { It can be seen that } \\
& \varepsilon(t)=\Gamma_{1} e(t)+\Gamma_{2} \tilde{h}(t)+\Delta(t) \\
& \quad \text { From } \mid \omega(z, u(t), F \mid \leq \delta \text {, it can be verified that } \\
& \|\Delta(t)\|=\left\|\int_{a}^{b} \sigma(z) \omega(z, u, F) d z\right\| \leq \alpha \quad \forall \alpha=\delta \int_{a}^{b} \sigma(z) d z
\end{aligned}
$$

$\mathrm{E}$ is a singular matrix,hence exist two orthogonal matrices $\mathrm{U}$ and $\mathrm{V}$ such that

$$
U E V=\left[\begin{array}{ll}
\Sigma & 0 \\
0 & 0
\end{array}\right]
$$

where $\Sigma=\operatorname{diag}\left(\lambda_{i}\right)$ and $\lambda_{\mathrm{i}}>0$ are the singular values of singular matrix $\mathrm{E}$.

As shown in reference [7] and [8],Denote

$U\left(A-L \Gamma_{1}\right) V=\left[\begin{array}{ll}A_{11} & A_{12} \\ A_{21} & A_{22}\end{array}\right], \quad\left[\begin{array}{l}e_{1}(t) \\ e_{2}(t)\end{array}\right]=V^{-1} e(t)$

$U G=\left[\begin{array}{ll}G_{1} & G_{2}\end{array}\right]^{T}, U L \Gamma_{2}=\left[\begin{array}{ll}H_{1} & H_{2}\end{array}\right], U L=\left[\begin{array}{ll}L_{1} & L_{2}\end{array}\right]^{T}$,

Assumption 1. If $\mathrm{A}_{22}$ is invertible,then the following inequality

$$
\frac{\left\|A_{22}\right\|}{K\left(A_{22}\right)}>\delta_{1}\left\|G_{2}\right\|+\delta_{2}\|C\|\left\|H_{2}\right\|
$$

holds, where $\mathrm{K}\left(\mathrm{A}_{22}\right)$ is the condition number of $\mathrm{A}_{22}$.

In the absence of F, Eq.(4) is transformed into

$\left\{\begin{array}{c}\Sigma \dot{e}_{1}(t)=A_{11} e_{1}(t)+A_{12} e_{2}(t)+G_{1} \tilde{g}(t)-H_{1} \tilde{h}(t)-L_{1} \Delta(t) \\ 0=A_{21} e_{1}(t)+A_{22} e_{2}(t)+G_{2} \tilde{g}(t)-H_{2} \tilde{h}(t)-L_{2} \Delta(t)\end{array}\right.$

First, when there is no fault, our objective is to find $\mathrm{L}$ such that the system (12) is stable, which can be formulated in the following theorem.

Theorem 1. For the parameters $\gamma>0$ and $\kappa_{\mathrm{i}}>0,(\mathrm{i}=1,2$

), if there exist matrices $\mathrm{R}$ and $\mathrm{P}$ with $\mathrm{P}$ being nonsingular,satisfying

$$
\begin{aligned}
& E^{T} P=P^{T} E \geq 0 \\
& {\left[\begin{array}{ccccc}
\Pi & P^{T} G & R \Gamma_{2} & C^{T} U_{1}^{T} & U_{2} \\
* & -\frac{1}{\kappa_{1}} & 0 & 0 & 0 \\
* & * & -\frac{1}{\kappa_{2}} & 0 & 0 \\
* & * & * & -\kappa_{1} & 0 \\
* & * & * & * & -\kappa_{2}
\end{array}\right]<0}
\end{aligned}
$$

where $\Pi=A^{T} P+P^{T} A-R \Gamma_{1}-\Gamma_{1}^{T} R^{T}+\gamma I$, then in the absence of $\mathrm{F}(\mathrm{x}, \mathrm{u})$, the error system (11) with gain $\mathrm{L}=\mathrm{P}^{-\mathrm{T}} \mathrm{R}$ is asymptotically stable.

Proof. Substituting Eq.(10) and (11) into Eq.(13), it can be seen that $\Sigma P_{11}=P_{11}^{T} \Sigma^{T}>0$ and $\mathrm{P}_{12}=0 . \mathrm{A}_{22}$ and $\mathrm{P}_{22}$ are inveritible.

Define the Lyapunov candidate function as follows

$$
\begin{aligned}
V(t) & =e^{T}(t) E^{T} P e(t)+\kappa_{1} \int_{0}^{t}\left[\left\|U_{1} C e(s)\right\|^{2}-\|\tilde{h}(s)\|^{2}\right] d s+\kappa_{2} \int_{0}^{t}\left[\left\|U_{2} e(s)\right\|^{2}-\|\tilde{g}(s)\|^{2}\right] d s \\
& =\dot{e}^{T}(t) E^{T} P e(t)+e^{T}(t) E^{T} P \dot{e}(t)+\kappa_{1} \int_{0}^{t}\left[\left\|U_{1} C e(s)\right\|^{2}-\|\tilde{h}(s)\|^{2}\right] d s+\kappa_{2} \int_{0}^{t}\left[\left\|U_{2} E e(s)\right\|^{2}-\|\tilde{g}(s)\|^{2}\right] d s
\end{aligned}
$$

Along the trajectories of (12) in the absence of F,it can be shown that

$$
\begin{aligned}
\dot{V}(t)= & e^{T}(t)\left[\left(A-L \Gamma_{1}\right)^{T} P+P^{T}\left(\left(A-L \Gamma_{1}\right)\right] e(t)+2 e^{T}(t) P^{T} G \tilde{g}(t)-2 e^{T}(t) R \Gamma_{2} \tilde{h}(t)\right. \\
& -2 e^{T}(t) P^{T} L_{2} \Delta(t)+\kappa_{1}\left[\left\|U_{1} C e(s)\right\|^{2}-\|\tilde{h}(s)\|^{2}\right]+\kappa_{2}\left[\left\|U_{2} e(s)\right\|^{2}-\|\tilde{g}(s)\|^{2}\right]
\end{aligned}
$$

Since

$$
\begin{aligned}
& 2 e^{T}(t) P^{T} G \tilde{g}(t) \leq \frac{1}{\kappa_{1}} e^{T}(t) P^{T} G G^{T} P e(t)+\kappa_{1} \tilde{g}^{T}(t) \tilde{g}(t) \\
& -2 e^{T}(t) P^{T} L \Gamma_{2} \tilde{h}(t) \leq \frac{1}{\kappa_{2}} e^{T}(t) P^{T} L \Gamma_{2} \Gamma_{2}^{T} L^{T} P+\kappa_{2} \tilde{h}^{T}(t) h(t)
\end{aligned}
$$

Using Assumptions 1 and 2, then we can get that

$$
\begin{gathered}
\dot{V}(t) \leq e^{T}(t)\left[\left(A-L \Gamma_{1}\right)^{T} P+P^{T}\left(A-L \Gamma_{1}\right)+\frac{1}{\kappa_{1}} P^{T} G G^{T} P+\frac{1}{\kappa_{2}} P^{T} L \Gamma_{2} \Gamma_{2}^{T} L^{T} P\right. \\
\left.+\kappa_{1} C^{T} U_{1}^{T} U_{1} C+\kappa_{2} U_{2}^{T} U\right] e(t)-2 e^{T}(t) P^{T} L_{2} \Delta(t)
\end{gathered}
$$


Thus, under (14), it can be seen that

$$
\begin{aligned}
\dot{V}(t) & <-\gamma\|e(t)\|^{2}--2 e^{T}(t) P^{T} L_{2} \Delta(t) \\
& \leq-\gamma\|e(t)\|^{2}+2 \alpha\|e(t)\|\|R\|
\end{aligned}
$$

Therefore, it can be claimed that $\left\|e_{1}(t)\right\| \leq \eta_{1}=\max \left\{\left\|e_{1}(0)\right\|, 2 \gamma^{-1} \alpha\|R\|\right\}$

As before, $\mathrm{A}_{22}$ is inveritible, by Eq.(12) and assumption 1,2 and 3, we can calculated that

$$
\begin{aligned}
\left\|e_{2}(t)\right\| \leq & \left\|A_{22}^{-1}\right\|\left\|A_{21} e_{1}(t)+G_{2} \tilde{g}(t)-H_{2} \tilde{h}(t)-L_{2} \Delta(t)\right\| \\
& =\frac{K\left(A_{22}\right)\left\|A_{21} e_{1}(t)+G_{2} \tilde{g}(t)-H_{2} \tilde{h}(t)-L_{2} \Delta(t)\right\|}{\left\|A_{22}\right\|} \\
& \leq \frac{\left(\left\|A_{21}\right\|+\delta_{1} G_{2}+\delta_{2}\left\|H_{2}\right\|\|C\|\right) \eta_{1}+\left\|L_{2}\right\| \alpha}{\delta_{1}\left\|G_{2}\right\|+\delta_{2}\|C\|\left\|H_{2}\right\|}
\end{aligned}
$$

It can be seen that

$$
e_{2}(t) \leq \eta_{2}=\max \left\{\left\|e_{2}(0)\right\|, \frac{\left(\left\|A_{21}\right\|+\delta_{1} G_{2}+\delta_{2}\left\|H_{2}\right\|\|C\|\right) \eta_{1}+\left\|L_{2}\right\| \alpha}{\delta_{1}\left\|G_{2}\right\|+\delta_{2}\|C\|\left\|H_{2}\right\|}\right\}
$$

From Eq.(15) and (16), we can seen that the error system is stable.

Theorem 1 presents a necessary condition for fault detection. In order to detect $F$, we select $\varepsilon(t)$ as residual signal and propose the following result to determine the threshold.

\section{B. Observer-based fault diagnosis}

After the fault is detected based upon the results in section 3.1, the fault diagnosis need to be carried out in order to estimate the size of fault F.when a fault occurs, we construct the following adaptive observer.

$$
\left\{\begin{array}{l}
E \dot{\hat{x}}(t)=A \hat{x}(t)+G g(\hat{x}(t))+D u(t)+L \varepsilon(t)+T \hat{W} S(\hat{x}, u) \\
\varepsilon(t)=\int_{a}^{b} \sigma(z)[\sqrt{\gamma(z, u(t), F)}-\sqrt{\hat{\gamma}(z, u(t), F)}] d z \\
\sqrt{\hat{\gamma}(z, u(t), F)}=B(z) C \hat{x}(t)+h(C \hat{x}(t)) b_{n}(z)
\end{array}\right.
$$

The system (4) can be rewritten as

$\{E \dot{x}(t)=A x(t)+G g(x(t))+D u(t)+T W S(x, u)+\theta(x, u)$

$V(t)=C x(t)$

By

$\tilde{W}=W-\hat{W}, \hat{S}=S(x, u)-S(\hat{x}, u), \theta_{1}=T W \hat{S}+\theta$ defining $\left\|\theta_{1}\right\| \leq \vartheta$, the estimation system can be described as $E \dot{e}(t)=\left(A-L \Gamma_{1}\right) e(t)+G \tilde{g}(t)-L \Gamma_{2} \tilde{h}(t)-L \Delta(t)+T \tilde{W} S(\hat{x}, u)+\theta_{1}$

Then, an adaptive fault estimation algorithm is presented by the following theorem.

Theorem 2 For the parameters $\kappa_{\mathrm{i}}>0(\mathrm{i}=1,2)$, if there exist matrices $\mathrm{R}, \mathrm{P}$ with $\mathrm{P}$ being non-singular,and $\beta>$ 0, such that the following LMI holds:

$$
\begin{aligned}
E^{T} P & =P^{T} E \geq 0 \\
M & {\left[\begin{array}{ccccc}
\Pi & \frac{1}{\kappa_{1}} P^{T} G & R \Gamma_{2} & C^{T} U_{1}^{T} & U_{2} \\
* & -\frac{1}{\kappa_{1}} & 0 & 0 & 0 \\
* & * & -\frac{1}{\kappa_{2}} & 0 & 0 \\
* & * & * & -\kappa_{1} & 0 \\
* & * & * & * & -\kappa_{2}
\end{array}\right]<0 }
\end{aligned}
$$

Where $\Pi=A^{T} P+P^{T} A-R \Gamma_{1}-\Gamma_{1}^{T} R^{T}+\gamma I$, Then the error system (19) with gain $\mathrm{L}=\mathrm{P}^{-\mathrm{T}} \mathrm{R}$ is stable and the fault estimation algorithm is as

$$
\dot{\hat{W}}=-\pi T^{T} \operatorname{Pe}(t) S^{T}(\hat{x}, u)
$$

Proof The Lyapunov candidate function can be choose as

$V(t)=e^{T}(t) E^{T} P e(t)+\operatorname{tr}\left\{\tilde{W}^{T} \pi^{-1} \tilde{W}\right\}+\kappa_{1} \int_{0}^{t}\left[\left\|U_{1} C e(s)\right\|^{2}-\|\tilde{h}(s)\|^{2}\right] d s+\kappa_{2} \int_{0}^{t}\left[\left\|U_{2} e(s)\right\|^{2}-\|\tilde{g}(s)\|^{2}\right] d s$

By Eq.(19) and using $\dot{\tilde{W}}=\dot{\hat{W}}=-\pi T^{T} \operatorname{Pe}(t) S^{T}(\hat{x}, u)$, we can obtain

$\dot{V}(t)=\dot{e}^{T}(t) E^{T} P e(t)+e^{T}(t) E^{T} P \dot{e}(t)+2 \operatorname{tr}\left\{\dot{\tilde{W}}^{T} \pi^{-1} \tilde{W}\right\}$ $+\kappa_{1}\left(e^{T}(t) C^{T} U_{1}^{T} U_{1} C e(t)-\tilde{h}^{T}(t) h(t)\right)+\kappa_{2}\left(e^{T}(t) U_{2}^{T} U_{2} e(t)-\tilde{g}^{T}(t) g(t)\right)$ $=e^{T}(t)\left[P^{T} A+A^{T} P-R \Gamma_{1}-\Gamma_{1}^{T} R^{T}\right] e(t)+2 e^{T}(t) P^{T} G \tilde{g}(t)-2 e^{T}(t) R \Gamma_{2} \tilde{h}(t)$ $-2 e^{T}(t) R \Delta(t)+2 e^{T}(t) P T \tilde{W} S(\hat{x}, u)+2 e^{T}(t) P \theta_{1}-2 \operatorname{tr}\left\{S(\hat{x}, u) e^{T}(t) P^{T} T \tilde{W}\right\}$ $+\kappa_{1}\left(e^{T}(t) C^{T} U_{1}^{T} U_{1} C e(t)-\tilde{h}^{T}(t) h(t)\right)+\kappa_{2}\left(e^{T}(t) U_{2}^{T} U_{2} e(t)-\tilde{g}^{T}(t) g(t)\right)$

It is noted that

$e^{T}(t) P T \tilde{W} S(\hat{x}, u)=\operatorname{tr}\left\{e^{T}(t) P T \tilde{W} S(\hat{x}, u)\right\}=2 \operatorname{tr}\left\{S(\hat{x}, u) e^{T}(t) P^{T} T \tilde{W}\right\}$

Then, we can obtained that

$$
\begin{aligned}
\dot{V}(t) \leq & e^{T}(t)\left[P^{T} A+A^{T} P-R \Gamma_{1}-\Gamma_{1}^{T} R^{T}+\frac{1}{\kappa_{1}} P^{T} G G^{T} P+\frac{1}{\kappa_{2}} R \Gamma_{2} \Gamma_{2}^{T} R^{T}\right. \\
& \left.+\kappa_{1} C^{T} U_{1}^{T} U_{1} C+\kappa_{2} U_{2}^{T} U\right] e(t)-2 e^{T}(t) R \Delta(t)+2 e^{T}(t) P \theta_{1} \\
\leq & e^{T}(t) \mathrm{M} e(t)-2 e^{T}(t) R \Delta(t)+2 e^{T}(t) P \theta_{1} \\
\leq & -\beta e^{T}(t) e(t)-2 e^{T}(t) R \Delta(t)+2 e^{T}(t) P \theta_{1} \\
= & -\frac{\beta}{2} e^{T}(t) e(t)-\frac{\beta}{4}\left[e^{T}(t) e(t)+\frac{8}{\beta} e^{T}(t) R \Delta(t)\right]-\frac{\beta}{4}\left[e^{T}(t) e(t)-\frac{8}{\beta} e^{T}(t) P \theta_{1}\right]
\end{aligned}
$$

Since

$$
\begin{aligned}
& \frac{8}{\beta} e^{T}(t) P \theta \leq e^{T}(t) e(t)+\frac{16}{\beta^{2}} \theta^{T} P^{T} P \theta_{1} \\
& -\frac{8}{\beta} e^{T}(t) R \Delta(t) \leq e^{T}(t) e(t)+\frac{16}{\beta^{2}} \Delta^{T} R^{T} R \Delta
\end{aligned}
$$

It is noted that

$$
\dot{V}(t) \leq-\frac{\beta}{2}\|e(t)\|^{2}+\frac{4 \varsigma_{1}}{\beta} \vartheta^{2}+\frac{4 \varsigma_{2}}{\beta} \alpha^{2}
$$

Where $\varsigma_{1}=\lambda_{\text {max }}\left(P^{T} P\right), \varsigma_{2}=\lambda_{\text {max }}\left(R^{T} R\right)$ are the maximum eigenvalue of $\mathrm{P}^{\mathrm{T}} \mathrm{P}$ and $\mathrm{R}^{\mathrm{T}} \mathrm{R}$.

In the presence of $\mathrm{F}$,the following inequality can be obtained that

$$
\left\|e_{1}(t)\right\|^{2} \leq \tau_{1}=\min \left\{\left\|e_{1}(0)\right\|^{2}, \frac{8}{\beta^{2}}\left(\lambda_{\max }\left(P^{T} P\right) \vartheta^{2}+\lambda_{\max }\left(R^{T} R\right) \alpha^{2}\right)\right\}
$$

From Eq.(12), we have

$$
\left\|e_{2}(t)\right\| \leq \tau_{2}=\max \left\{\left\|e_{2}(0)\right\|, \frac{\left(\left\|A_{21}\right\|+\delta_{1} G_{2}+\delta_{2}\left\|H_{2}\right\|\|C\|\right) \sqrt{\tau_{1}}+\left\|L_{2}\right\| \alpha}{\delta_{1}\left\|G_{2}\right\|+\delta_{2}\|C\|\left\|H_{2}\right\|}\right\}
$$

Eq. (19) with diagnosis observer Eq.(16) based on gain $\mathrm{L}=\mathrm{P}^{-\mathrm{T}} \mathrm{R}$ is stable and the estimation error satisfies

$$
\|e(t)\|^{2} \leq \tau_{1}+\tau_{2}^{2}
$$

If $\|e(t)\|^{2}>\tau_{1}+\tau_{2}^{2}$ is satisfied,which indicates that $\left\|e_{1}(t)\right\|$ or $\left\|e_{2}(t)\right\|$ is larger than $\tau_{1}$ or $\tau_{2}$. it can be seen that that fault occurs in this case. Eq.(25) means that the estimated error be guaranteed to be small if we selects suitable design parameters. This implies that the estimated error can be made arbitrarily small by choosing the suitable design parameters $\beta, \varsigma_{1}, \varsigma_{2}$ and L. 


\section{CONCLUSION}

In this paper,a new FDD method is investigated for singular non-Gaussian stochastic system. It is developed from the technology of PDFs, which is modeled by a square root B-spline expansion. Based on LMI techniques, the complexity FDD problem of singular non-Gaussian stochastic system is transformed into the classical nonlinear FDD problem by introducing the tuning parameter,the corresponding estimation error system is guaranteed to be stable.

\section{REFERENCES}

[1] M.Basseville,I.Nikiforov.Fault isolation for diagnosis: nuisance rejection and multiple hypothesis testing. Ann. Rev. Contr., 2002, 26:189-202

[2] Lei Guo. Crespo ,J. Q. Sun.Non-linear stochastic control via stationary response design.Prob. Eng. Mech, 2003,18:73-86.

[3] Jinglin Zhou,Donghua Zhou.Distribution function tracking filter design using hybrid characteristic functions.Automatica, 2010,46:101-109.

[4] Bo Shen,Huisheng Shu, Hœfiltering for nonlinear discrete-time stochastic systems with randomly varying sensor delays. Automatica ,2009,45:1032-1037.

[5] Ali.Okatan,Chingiz,Hajiyev.Fault detection in sensor information fusion Kalman filter. Int. J. Electron. Commun. (AEÜ), 2009,63:762-768.

[6] R. Isermann,P. Balle.Trends in the application of model based fault detection and diagnosis of technical process.in Proc. 13th World Congr. Int. Federation Automatic Control (IFAC'96), San Francisco,CA, 1996, N:1-12.

[7] Tao Li,Yingchao Zhang. Fault detection and diagnosis for stochastic systems via output PDFs. Journal of the Franklin Institute, 2011,348:1140-1152.

[8] Zineb Simeu-Abazi, Maria Di Mascolo, Michal Knotek. Fault diagnosis for discrete event systems: Modelling and verification. Reliability Engineering and System Safety, 2010,95:369-378.

[9] Karim Salahshoor, Mojtaba Kordestani, Majid S. Khoshro. Fault detection and diagnosis of an industrial steam turbine using fusion of SVM(support vector machine) and ANFIS (adaptive neuro-fuzzy inference system) classifiers. Energy, 2010,35:5472-5482.

[10] Hui Li ,Yuping Zhang,Haiqi Zheng. Gear fault detection and diagnosis under speed-up condition based on order cepstrum and radial basis function neural network.Journal of Mechanical Science and Technology, 2009,23:2780 2789.

[11] Lei Guo, Hong Wang. Fault detection and diagnosis for general stochastic systems using B-spline expansions and nonlinear filters. IEEE Trans Circuits Syst I.2005; 52(8): 1644-52.

[12] Hong Wang. Applying observer based FDI techniques to detect faults in dynamic and bounded stochastic distributions. Int J Control. 2000; 73(15):1424-36.

[13] [13]Hong Wang. Bounded dynamic stochastic distributions modeling and control.Springer-Verlag, 2000.

[14] ao Li , Yang Yi , Lei Guo , Hong Wang. Delay-dependent fault detection and diagnosis using B-spline neural networks and nonlinear filters for time-delay stochastic systems. Neural Comput \& Applic, 2008,17:405-411.

[15] LiNa Yao , Hong WANG. Robust fault diagnosis for non-Gaussian stochastic systems based on the rational square-root approximation model. Sci China Ser F-Inf Sci, 2008,51 (9): 1281-1290.

[16] Zakwan Skaf,Hong Wang,Lei Guo.Fault tolerant control based on stochatic distribution via RBF neural network.Journal of systems Engineering and Electrics,2011,22(1):63-69. 